\title{
EcoHealth: Envisioning and Creating a Truly Global Transdiscipline
}

Transdisciplinarity or transdisciplinary thinking (TD) employs perspectives and methods that transcend traditional disciplinary boundaries and engage both researchers and practitioners in addressing real-world problems. TD requires the team members to share roles and systematically cross disciplinary boundaries. The primary purpose of this approach is to pool and integrate the team expertise so that more efficient and comprehensive assessment and intervention may be provided in a determined field. The communication style in TD involves continuous give-and-take among all members on a regular, planned basis. Assessment, intervention, and evaluation are carried out jointly. TD brings together academic experts, field practitioners, community members, research scientists, political leaders, and business owners among others to solve some of the pressing problems facing the world, from the local to the global, requiring the natural and social sciences to address the irrevocably linked problem of the ecology and health of species and ecosystems. A previous editorial drew attention to this journal's transdisciplinary mission and described the current understanding of what constitutes TD (Wilcox and Kueffer, 2008).

The real-world problems that provoked the launch of the scientific journal EcoHealth in 2004 are the converging global challenges of climate change, biodiversity loss, land use change, emerging infectious diseases, global toxification, ecological health, and sustainability. Implicit in their characterization as "real-world" and "global," and consistent with "participation" as a cornerstone of TD, is that EcoHealth should represent a globally inclusive community of researchers and practitioners.

This of course means the journal content should reflect this global community diversity in terms of geography, nationality, as well as scientific and cultural perspectives. Yet we have a long way to become a truly diverse and global journal. Perusal of this and most issues of EcoHealth demonstrate that the contributions to the journal are overwhelmingly (more than 75\%) by authors from institutions in so-called developed nations. We use "developed" with caution here, since it is a construct based on the prevailing model of industrial economic development that represents anything but an improvement in the health and well-being for most of the world's human population. Not only in less developed countries but also in developed ones, the incidence of obesity, diabetes, cardiovascular syndromes, and related chronic diseases reflect lifestyles and diet increasingly disconnected from nature.

Having focused during the past 4 years on getting EcoHealth "up and running," including encouraging a sufficient flow of quality submissions and securing indexing on Web of Science, this year and next represents a pivotal time for the journal. The journal recently succeeded in being added to PubMed and Medline indexing systems; our newly created organization, the International Society for Ecology and Health (IAEH), has achieved stature as co-sponsor of the EcoHealth 2008 Forum to be held in Merida, Yucatan, Mexico, December 1-5, 2008 (http://www.ecohealth.net). We feel these events, and the ascent of EcoHealth as a paradigm for understanding and addressing the intertwined global challenges discussed above, compel us to foster the next step in the journal's evolution toward becoming global.

EcoHealth was born of desire to begin truly integrating disciplines and the urgent need to address the rapid deterioration of the planet. This integration requires commitment not only from government agencies, universities, and other organizations, but eventually the need to generate 
new international structures, global networks including regionally-based communities, and even IAEH chapters in which EcoHealth can serve as a truly global professional outlet bridging the "developed" and "developing" world divide. EcoHealth already has come a long way toward filling the need to link areas of research and practice in conservation medicine, human health, and ecosystem sustainability. The journal and supporters of this field based mainly in the developed North led by default in creating the IAEH. Our 2nd biennial meeting, Ecohealth Forum 2008 in Mérida, presents a critical opportunity to expand the Association's base in Africa, Asia, and Latin America, making the journal and association truly global.

These regions have, by far, most of the world human population, poverty, biodiversity, and environmental health challenges. On the other hand, on a per capita basis, the developed world consumes a vastly disproportionate amount of the global natural resources and produces a similarly disproportionate share of the world's waste and pollution. EcoHealth must orient itself toward research and practice that accounts for these global disparities and address them in a human development context. At this pivotal time in our environmental history, these problems urgently require TD approaches that are truly participatory-geographically, socially, and culturally. The need for global capacity in EcoHealth research networks and communities of practice capable of breaking down institutional barriers and building collaborative bridges-and being more candid about the disparities-is critical to taking us to the next level. When $75 \%$ of the submissions to EcoHealth come from tropical and less economically supported countries, perhaps part of our mission may have been achieved.

Alonso Aguirre

Conservation Medicine Program

Wildlife Trust, New York, NY, USA

e-mail: aguirre@wildlifetrust.org.

Bruce A. Wilcox

John A. Burns School of Medicine

University of Hawaii at Manoa, Honolulu, HI, USA

\section{REFERENCE}

Wilcox B, Kueffer C (2008) Transdisciplinarity in EcoHealth: status and future prospects. EcoHealth 5:1-3

Published online: December 3, 2008 